\title{
La cycline T1 est nécessaire à l'activation par Tat de la transcription du VIH
}

$\mathbf{P}$ armi les 15 protéines codées par le VIH, virus de l'immunodéficience humaine, la protéine Tat est l'une des plus étudiées (pour des revues, voir $[1,2])$. L'activation de la transcription du génome viral a été la première des fonctions caractérisées de Tat. La protéine Tat influe également sur l'expression de quelques gènes cellulaires, soit en activant (facteur nécrosant des tumeurs ou TNF, interleukines-2 et -6), soit en réprimant leur transcription (superoxyde dismutase dépendant du manganèse ou MnSOD, et complexe majeur d'histocompatibilité ou CMH de classe I). La protéine Tat s'associe à l'ARN polymérase II et à de très nombreux facteurs de transcription. Comme nous allons le voir, des travaux récents montrent que les interactions de Tat avec l'ARN viral TAR (trans activating region), avec l'histoneacétyl-transférase p300 et avec la cycline $\mathrm{T} 1$ jouent un rôle clé dans l'activation de la transcription virale.

\section{Le repliement de l'ARN TAR précède l'activation de la transcription du VIH par Tat}

La protéine Tat stimule très fortement la transcription à partir du promoteur situé dans les répétitions terminales (LTR, long terminal repeat) du $\mathrm{VIH}$ (figure 1). Des séquences d'ADN placées en amont (sites SP1, boîte TATA, TAR et, dans certaines cellules, sites NFKB) ou en aval (TAR) du site de démarrage de la transcription sont indispensables à cette activation (pour des revues, voir [3, 4]). Deux types de transcrits sont engendrés à partir de ce promoteur: des transcrits «courts» non adénylés d'environ 60 nucléotides, et des transcrits «longs» polyadénylés, couvrant l'ensemble du génome viral [5]. La protéine Tat stimule très fortement la formation des transcrits «longs» aux dépens des transcrits «courts». L'action de Tat est postérieure au recrutement de la TBP (TATA-binding protein) sur le promoteur [6]. La transcription de la région TAR, qui s'étend sur les 50 premiers nucléotides du transcrit viral, est un préalable à une interaction TAR/Tat et donc à l'activation par Tat. Cette activation dépend du repliement de l'ARN TAR en une structure tridimensionnelle reconnue par Tat. Après avoir transcrit 62 nucléotides, l'ARN polymérase II marquerait une pause avant que l'ARN naissant puisse se replier pour former la structure «TAR» [7]. En l'absence de Tat, la polymérase aurait ensuite tendance à interrompre sa progression en produisant des transcrits « courts».

D'après un modèle proposé par Philip Sharp au début des années 1990, Tat modifierait les propriétés de l'ARN polymérase II déjà engagée dans la transcription sur le promoteur pour en favoriser la progression sur la matrice d'ADN (élongation de la transcription) et permettre la synthèse d'ARN viraux complets. Tat resterait associée à l'ARN polymérase II pendant l'élongation de la transcription [8]. D'autres modèles d'activation par Tat ont suggéré son action sur le démarrage ou le re-démarrage de la transcription. Cependant, c'est au niveau de l'élongation que l'intervention de Tat est actuellement la mieux établie grâce à un ensemble de travaux reposant sur: (1) une approche biochimique, ayant conduit à l'identification de protéines cellulaires se fixant sur la protéine Tat recombinante et/ou permettant l'activation de la transcription par Tat dans un test in vitro; (2) une approche génétique, tirant profit de la faible efficacité de l'activation par Tat dans des cellules de rongeurs.

\section{Des histone-acétyltransférases s'associent à Tat}

Les nucléosomes s'opposent à la progression de l'ARN polymérase II. Or, l'activation du LTR du VIH s'accompagne du déplacement de nucléosomes et elle est stimulée par les inhibiteurs d'histone-désacétylases [9]. Cette observation a conduit plusieurs équipes à rechercher si des histoneacétyltransférases ne s'associaient pas avec Tat. Tip60 a été isolée par la technique du double-hybride [10, 11]. D'autres histone-acétyltransférases, CBP, p300 et P/CAF s'associent in vitro à la protéine Tat recombinante et leur surexpression stimule la transcription dirigée par le promoteur du VIH [12-14]. Ainsi, dans les cellules murines, l'activation par Tat du LTR du VIH est très peu efficace, mais elle est très fortement augmentée si l'on surexprime p300 dans ces cellules. L'inefficacité de Tat dans les cellules de rongeurs pourrait donc être en partie attribuée à la très faible quantité de p300 présente dans ces cellules. A l'inverse, d'autres histoneacétyltransférases, par exemple TAF250 associée à la TBP, peuvent, lorsqu'elles interagissent avec Tat, inhiber l'expression de gènes cellulaires codant pour les molécules de classe I du complexe majeur d'histocompatibilité [15].

\section{La phosphorylation de l'ARN polymérase II stimulée par Tat} active l'élongation de la transcription

Le domaine carboxy-terminal (CTD) de la plus grande sous-unité de l'ARN polymérase II est un intermédiaire obligatoire pour l'activation de la transcription des gènes de classe II (m/s 1998, $\left.n^{\circ} 2, p .167\right)$. L'ARN polymérase IIA, lorsqu'elle n'est pas phosphorylée sur le domaine carboxy-terminal, s'intègre aux com- 


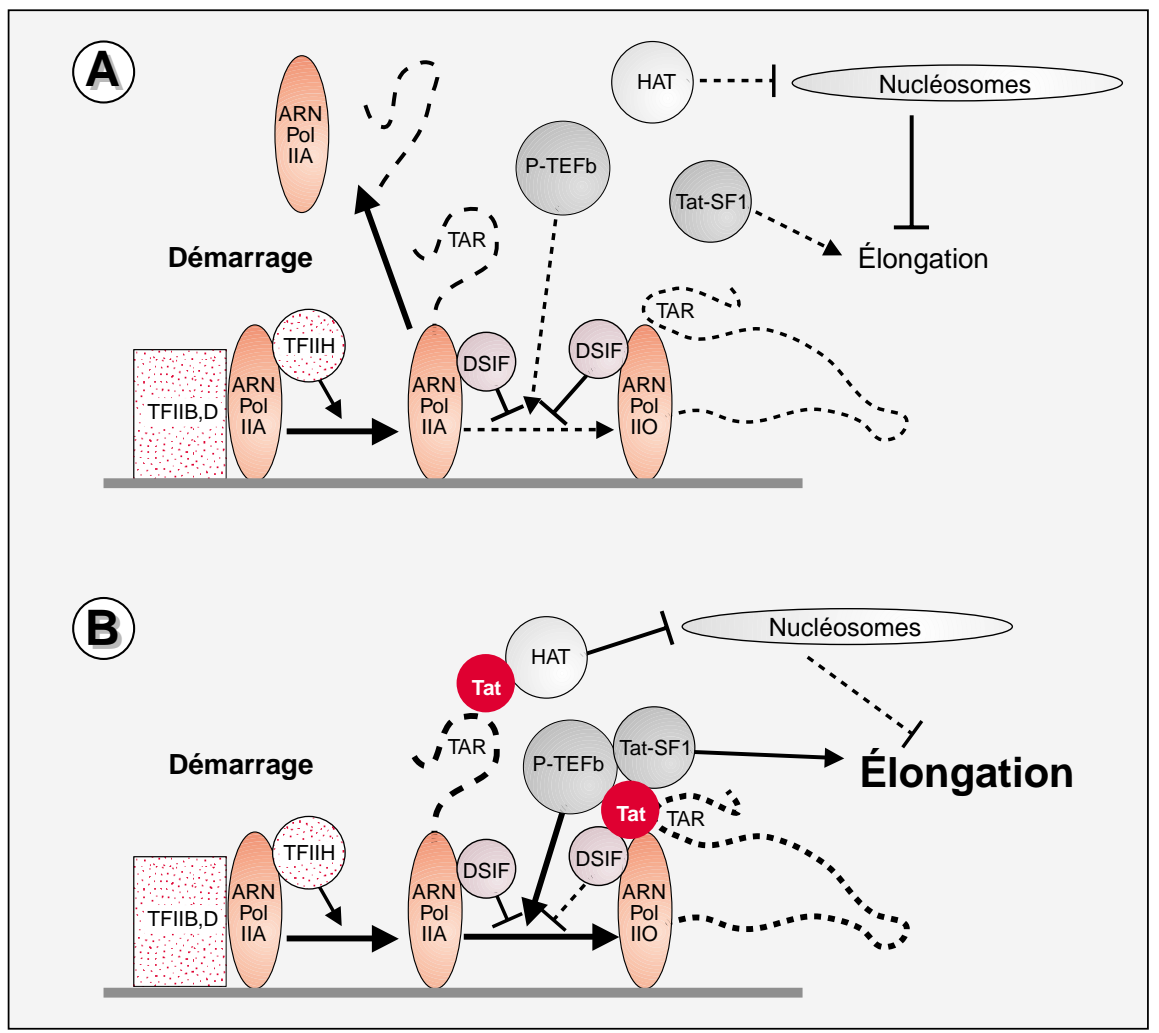

Figure 1. La protéine Tat provoque la convergence de plusieurs activateurs de la transcription sur le promoteur du VIH. La région TAR (à l'extrémité 5' du transcrit viral) est transcrite par l'ARN polymérase II hypophosphorylée (ARN Pol IIA). Le facteur TFIIH s'intègre au complexe de prédémarrage de la transcription avec le TFIID et le TFIIB. Le TFIIH est relâché au bout de quelques dizaines de nucléotides, avant la synthèse complète de l'ARN TAR. A. En I'absence de Tat, le DSIF demeure associé à I'ARN PollA, ce qui provoque le départ précoce de la polymérase hors de sa matrice ADN et la formation de transcrits "courts". B. La protéine Tat facilite l'assemblage de nombreux facteurs généraux de la transcription sur le génome viral et reste associée à I'ARN polymérase pendant l'élongation de la transcription. Un complexe associant Tat à la cycline T1 du facteur P-TEFb se lie à I'ARN TAR. Cette reconnaissance hautement spécifique stimule la phosphorylation de la polymérase par le P-TEFb. Le P-TEFb phosphoryle aussi le Tat-SF1, un autre facteur activateur de l'élongation de la transcription. Par ailleurs, Tat inhibe la phosphatase FCP1 qui déphosphoryle I'ARN Pol IIO en ARN Pol IIA. L'action de DSIF est neutralisée par la phosphorylation de l'ARN polymérase II en ARN Pol IIO par le facteur P-TEFb, ce qui permet la synthèse des transcrits viraux complets. Parmi les nombreux facteurs recrutés par Tat, des histone-acétyltransférases (HAT) atténuent le frein opposé par les nucléosomes à l'avancement de la polymérase. Les interactions spécifiques qui se forment entre Tat et le P-TEFb ou entre Tat et I'histone-acétyltransférase p300, contribuent à l'écotropie du VIH.

plexes de prédémarrage de la transcription et commence à transcrire quelques dizaines de nucléotides. Puis la transcription avorte si le domaine carboxy-terminal n'est pas phosphorylé, ce qui empêche la pro1174 transcription, P-TEFb (positive transcription elongation factor) [18-21]. TFIIH (qui exerce d'autres activités que la phosphorylation) n'interviendrait qu'au démarrage de la transcription, avant le repliement de l'ARN TAR, alors que le P-TEFb, lui, ferait partie du complexe d'élongation de la transcription [22]. S'il est maintenant admis que l'activité CTD-kinase de P-TEFb est indispensable à l'activation du LTR du VIH par Tat, l'implication de la sousunité CDK7 de TFIIH reste controversée [23].

Non seulement Tat stimule la phosphorylation du domaine carboxy-terminal, mais elle en inhibe la déphosphorylation par la CTD-phosphatase dépendante du TFIIH [24]. L'une des fonctions de Tat serait donc de permettre l'élongation de la transcription en favorisant la phosphorylation du domaine carboxy-terminal.

La recherche de co-facteurs cellulaires nécessaires à l'activation transcriptionnelle par Tat-1 in vitro a conduit à l'isolement du facteur TatSF1 (Tat stimulating factor 1) [25-27]. Tat-SF1 est un substrat de CDK9, mais aussi un activateur général de l'élongation de la transcription, et serait impliqué dans l'épissage des ARN. La même démarche a permis l'identification du complexe protéique SPT4/SPT5, aussi appelé DSIF (DRB-sensitivity-inducing factor) [28]. Le complexe DSIF est un répresseur de la transcription, neutralisé par P-TEFb [29]. En demeurant associé à l'ARN polymérase IIA (non phosphorylée), DSIF empêcherait l'élongation de la transcription au-delà de la région TAR, ce qui provoquerait le départ précoce de la polymérase hors de sa matrice d'ADN et la formation de transcrits «courts». La phosphorylation du domaine carboxy-terminal de la polymérase en ARN polymérase IIO par P-TEFb libérerait le répresseur DSIF et permettrait la transcription complète du génome viral.

La cycline $\mathrm{T} 1$ stabilise la liaison TAR/Tat et détermine l'écotropie de plusieurs lentivirus

Tat: CDK7 (cyclin dependent kinase 7) la kinase du facteur général de la transcription (et de la réparation de l'ADN) TFIIH $\left(m / s\right.$ 1995, $\left.n^{\circ} 6, p .879\right)$ $[16,17]$, et CDK9, la kinase du complexe activateur de l'élongation de la
Le VIH ne se réplique pas dans des cellules de rongeurs. Or, la protéine Tat active très faiblement la trans- 
cription du VIH dans ces cellules. L'implication de P-TEFb dans la stimulation par Tat in vivo est fortement étayée par le fait que l'expression de la cycline T1 humaine dans des cellules de rongeurs induit une stimulation du promoteur VIH par Tat dont l'efficacité égale celle que l'on observe dans des cellules humaines [30]. Dans le complexe $\mathrm{P}-\mathrm{TEFb}$, la kinase CDK9 est associée à l'une des cyclines $\mathrm{T}$ : T1, T2a ou T2b. A la différence de la cycline $\mathrm{T} 1$, les cyclines $\mathrm{T} 2 \mathrm{a}$ et $\mathrm{T} 2 \mathrm{~b}$ humaines n'influencent pas l'action de Tat. La cycline $\mathrm{T} 1$, dont le gène est cloné chez l'homme et chez la souris, possède une séquence primaire légèrement différente dans ces deux espèces. En particulier, l'acide aminé 261 est une cystéine dans la cycline T1 humaine et une tyrosine dans la cycline $\mathrm{T} 1$ murine. Les cyclines $\mathrm{T} 1$ humaines et murines interagissent toutes deux avec Tat, mais seul le complexe Tat/cycline T1 humain se lie fortement à l'ARN TAR et induit la transactivation de la transcription. Cette association augmente fortement l'affinité de Tat pour TAR, alors que l'ARN TAR n'influence pas l'interaction Tat/cycline T1. Le remplacement de la tyrosine 261 par une cystéine rend la cycline T1 murine aussi efficace que la cycline $\mathrm{T} 1$ humaine. Inversement, si la cystéine 261 de la cycline T1 humaine est remplacée par une tyrosine, l'activité de la cycline est abolie, et en conséquence la fixation de Tat à TAR et la transactivation. Ces expériences attribuent donc un rôle crucial à la cystéine 261 de la cycline T1 humaine. L'association de Tat à la cycline T1 humaine dépend d'ions $\mathrm{Zn}^{2+}$ qui pourraient être complexés par cette cystéine 261 et par les cystéines 22 et 37 de Tat-1 (du VIH-1) [20, 30].

Comment comprendre que l'expression de la cycline $\mathrm{T} 1$ humaine, d'une part, la surexpression de l'histone, acétylase p300, d'autre part, confèrent à Tat la capacité de transactiver le LTR du VIH dans des cellules de rongeurs? Deux hypothèses sont envisageables: soit deux voies d'activation parallèles existent, l'une utilisant la cycline T1/CDK9 et l'autre la p300; soit un complexe se forme entre quatre partenaires: TAR, Tat, cycline T1/CDK9 et p300, chaque partenaire contribuant à la cohésion de l'ensemble. L'association de la cycline $\mathrm{T} 1$ humaine à Tat pourrait renforcer l'affinité de p300 pour Tat et p300 deviendrait efficace même à faible concentration; réciproquement, la cycline T1 murine s'associerait moins bien à la protéine Tat isolée qu'au complexe p300/Tat dont la formation spontanée serait induite par la surexpression de p300.

Bien que l'expression de la cycline T1 humaine permette une transcription efficace du génome viral dans les cellules de rongeurs, elle ne suffit pas à la production de virus. L'intervention d'autres co-facteurs de Tat pourrait être nécessaire à d'autres étapes du cycle viral, telles que la rétrotranscription [31]. D'ailleurs, l'action de Tat ne se pas limite pas au compartiment nucléaire. Tat interagit aussi avec des protéines de la membrane plasmique [2] ou du cytoplasme [32].

Quoi qu'il en soit, il semble que l'importance des travaux mentionnés ci-dessus dépasse largement le cadre du VIH : l'activation transcriptionnelle de lentivirus très divergents, tels que le virus de l'anémie infectieuse équine (EIAV), fait également intervenir le recrutement du facteur PTEFb par une interaction Tat/ cycline T1 [33]. De façon générale, le fait que les inhibiteurs de CDK9 soient systématiquement des inhibiteurs de la phosphorylation du CTD et de la transcription laisse supposer que le $\mathrm{P}-\mathrm{TEFb}$ est nécessaire à la transcription de la plupart des gènes de classe II $[34,35]$

\section{RÉFÉRENCES}

1. Kingsman SM, Kingsman AJ. The regulation of human immunodeficiency virus type-1 gene expression. Eur J Biochem 1996; 240: 491-507.

2. Rubartelli A, Poggi A, Sitia R, Zocchi MR. HIV-1 Tat: a polypetide for all seasons. Immunol Today 1998; 19: 543-5.

3. Jones KA, Peterlin BM. Control of RNA initiation and elongation at the HIV-1 promoter. Annu Rev Biochem 1994; 63: 717-43.

4. Garcia JA, Gaynor R. The human immunodeficiency virus type-1 long terminal repeat and its role in gene expression. Progr Nucleic Acids Res 1994; 49: 157-96.
5. Morrison DJ, Pendergrast PS, Stavropoulos P, Colmenares SU, Kobayashi R, Hernandez N. FBI-1, a factor that binds to the HIV-1 inducer of short transcripts (IST), is a POZ domain protein. Nucleic Acids Res 1999; 27: 1251-62.

6. Xiao H, Lis JT, Jeang KT. Promoter activity at steps subsequent to TATA-binding protein recruitment. Mol Cell Biol 1997; 17: 6898-905.

7. Palangat M, Meier TI, Keene RG, Landick R. Transcriptional pausing at +62 of the HIV-1 nascent RNA modulates formation of the TAR RNA structure. Mol Cell 1998; $1: 1033-42$.

8. Keen N, Churcher MN, Karn J. Transfer of Tat and release of TAR RNA during the activation of the human immunodeficiency virus type-1 transcription elongation complex. EMBO J 1997; 16: 5260-72.

9. Van Lint C, Emiliani S, Ott M, Verdin E. Transcriptional activation and chromatin remodeling of the HIV-1 promoter in response to histone acetylation. $E M B O J 1996$; 15: 1112-20.

10. Kamine J, Elangovan B, Subramanian T, Coleman D, Chinnadurai, G. Identification of a cellular protein that specifically interacts with the essential cysteine region of the HIV-1 Tat transactivator. Virology 1996; 216: 357-66.

11. Yamamoto T, Horikoshi M. Novel substrate specificity of the histone acetyltransferase activity of HIV-1-Tat interactive protein Tip60. J Biol Chem 1997; 272: 30595-8.

12. Benkirane M, Chun RF, Xiao H, et al. Activation of integrated provirus requires histone acetyltransferase. P300 and P/CAF are coactivators for HIV-1 Tat. J Biol Chem 1998; 273 : 24898-905.

13. Hottiger MO, Nabel GJ. Interaction of human immunodeficiency virus type-1 Tat with the transcriptional coactivators p300 and CREB binding protein. J Virol 1998; 72: 8252-6.

14. Marzio G, Tyagi M, Gutierrez MI, Giacca M. HIV-1 Tat transactivator recruits p300 and CREB-binding protein histone acetyltransferases to the viral promoter. Proc Natl Acad Sci USA 1998; 95 : 13519-24.

15. Weissman JD, Brown JA, Howcroft TK, et al. HIV-1 Tat binds TAF $_{\text {II }} 250$ and represses $\mathrm{TAF}_{\mathrm{II}} 250$-dependent transcription of major histocompatibility class I gene. Proc Natl Acad Sci USA 1998; 95 : 11601-6.

16. Garcia-Martinez LF, Mavankal G, Neveu JM, Lane WS, Ivanov D, Gaynor RB. Purification of a Tat-associated kinase reveals a TFIIH complex that modulates HIV-1 transcription. EMBO J 1997; 16: 2836-50.

17. Cujec T, Okamoto H, Fujinaga K, et al. The HIV transactivator TAT binds to the CDK-activating kinase and activates the phosphorylation of the carboxy-terminal domain of RNA polymerase II. Genes Dev 1997; 11: 2645-57.

18. Zhu Y, Pe'ery T, Peng J, et al. Transcription elongation factor $\mathrm{P}-\mathrm{TEFb}$ is required for HIV-1 Tat transactivation in vitro. Genes Dev 1997; 11 : 2622-32. 


\section{RÉFÉRENCES}

19. Mancebo HS, Lee G, Flygare J, et al. $\mathrm{P}-\mathrm{TEFb}$ kinase is required for HIV Tat transcriptional activation in vivo and in vitro. Genes Dev 1997; 11 : 2633-44.

20. Wei P, Garber ME, Fang SM, Fischer WH, Jones KA. A novel CDK9-associated C-type cyclin interacts directly with HIV-1 Tat and mediates its high-affinity, loop-specific binding to TAR RNA. Cell 1998; 92: 451-62.

21. Fujinaga K, Taube R, Wimmer J, Cujec TP, Peterlin BM. Interactions between human cyclin $\mathrm{T}$, Tat, and the transactivation response element (TAR) are disrupted by a cysteine to tyrosine substitution found in mouse cyclin $\mathrm{T}$. Proc Natl Acad Sci USA 1999; 96: 1285-90.

22. Ping YH, Rana TM. Tat-associated kinase $(\mathrm{P}-\mathrm{TEFb})$ : a component of transcription preinitiation and elongation complexes. J Biol Chem 1999; 274: 7399-404.

23. Chen Dn Zhou Q. Tat activates human immunodeficiency virus type-1 transcriptional elongation independent of TFIIH kinase. Mol Cell Biol 1999; 19: 2863-71.

24. Marshall NF, Dahmus GK, Dahmus ME. Regulation of CTD phosphatase by HIV-1 Tat protein. J Biol Chem 1998; 273 : 31726-30.

25. Yan D, Perriman R, Igel H, Howe KJ, Neville M, Ares M Jr. CUS2, a yeast homolog of human Tat-SF1, rescues function of misfolded U2 through an unusual RNA recognition motif. Mol Cell Biol 1998; 18: 5000-9.
26. Zhou Q, Chen D, Pierstorff E, Luo K. Transcription elongation factor P-TEFb mediates Tat activation of HIV-1 transcription at multiple stages. EMBO J 1998; 17 : 3681-91.

27. Li XY, Green MR. The HIV-1 Tat cellular coactivator is a general transcription elongation factor. Genes Dev 1998; 12: 2992-6.

28. Wu-Baer F, Lane WS, Gaynor R. Role of the human homolog of the yeast transcription factor SPT5 in HIV-1 Tat-activation. $J$ Mol Biol 1998; 277: 179-97.

29. Yamaguchi Y, Takagi T, Wada T, et al. NELF, a multisubunit complex containing $\mathrm{RD}$, cooperates with DSIF to repress RNA polymerase II elongation. Cell 1999; 97 : 4151

30. Garber ME, Wei P, KewalRamani VN, et al. The interaction between HIV-1 Tat and human cyclin $\mathrm{T} 1$ requires zinc and a critical cysteine residue that is not conserved in the murine CycT1 protein. Genes Dev 1998; 12: 3512-27.

31. Harrich D, Ulich C, Garcia-Martinez LF, Gaynor RB. Tat is required for efficient HIV-1 reverse transcription. EMBO I 1997 ; $16: 1224-35$

32. Brand SR, Kobayashi R, Mathews MB. The Tat protein of human immunodeficiency virus type- 1 is a substrate and inhibitor of the interferon-induced, virally activated protein kinase, PKR. I Biol Chem 1997; $272: 8388-95$.
33. Bieniasz PD, Grdina TA, Bogerd HP, Cullen BR. Highly divergent lentiviral Tat proteins activate viral gene expression by a common mechanism. Mol Cell Biol 1999; 19: 4592-9.

34. Dubois MF, Nguyen VT, Bellier S, Bensaude $O$. Inhibitors of transcription such as 5,6-dichloro-1- $\beta$-D-ribofuranosyl benzimidazole (DRB) and isoquinoline sulfonamide derivatives (H-8 and $\left.\mathrm{H}-7^{*}\right)$, promote the dephosphorylation of the C-terminal domain (CTD) of RNA polymerase II largest subunit. J Biol Chem 1994; 269 : 13331-6.

35. Cassé C, Giannoni F, Nguyen VT, Dubois MF, Bensaude O. The transcriptional inhibitors, actinomycin D and $\alpha$-amanitin, activate the HIV-1 promoter and favor phosphorylation of the RNA polymerase II C-terminal domain. I Biol Chem 1999; 274 : 16097-106.

\section{Olivier Bensaude \\ Céline Cassé}

Laboratoire de régulation de l'Expression génétique, UMR Cnrs 8541, École normale supérieure, 46, rue d'Ulm, 75230 Paris Cedex 05, France.

\section{BRÈVES}

Rôle du co-récepteur CXCR4 ou CCR5 dans la cinétique d'infection par le VIS. L'infection de macaques par des souches virales chimériques (SHIV) entre le virus de l'immunodéficience humaine (VIH) et celui de l'immunodéficience simienne (VIS) a été établie pour fournir un modèle in vivo mimant l'infection par le VIH. Les premiers virus chimériques élaborés l'ont été à partir de souches virales utilisant le co-récepteur CXCR4 (X4) ou possédant un tropisme double X4-R5 (CCR5) ( $\mathrm{m} / \mathrm{s}$ 1997, $n^{\circ} 2$, p. 264), par substitution de la région tat, rev et $e n v$ du SIVmac239 par les régions correspondantes du clone viral VIH-1. Cependant, leur inoculation chez le macaque a provoqué une immunodéficience dont la cinétique est différente de celle qui est observée avec les isolats pathogènes de VIS ou de VIH qui utilisent exclusivement le co-récepteur R5. En effet, pendant la primoinfection, il y a une perte précipitée et persistante des cellules $\mathrm{T} \mathrm{CD}^{+}$ circulantes, sans disparition des lymphocytes du tractus intestinal, alors que dans les infections classiques, on observe une forte déplétion des cellules $\mathrm{T} \mathrm{CD}^{+}$du tractus gastrointestinal, avec une chute transitoire plus modeste des cellules $\mathrm{T} \mathrm{CD}^{+}$ circulantes. Afin de savoir si cette différence de cinétique est due à la différence de souche virale, Harouse et al. ont développé un virus chimérique spécifique du corécepteur R5 (SHIVSF162) [1]. L'inoculation de ce virus à des macaques provoque une infection primaire dont la cinétique est calquée sur celle des isolats spécifiques du co-récepteur R5. La différence de cinétique est certainement liée au fait que, dans le tractus intestinal, les cellules $\mathrm{T} \mathrm{CD}^{+}$mémoires, qui expriment préférentiellement le corécepteur R5, sont nombreuses alors que, dans les organes lymphoïdes et le sang, les cellules $\mathrm{T} \mathrm{CD} 4^{+}$expriment plus fortement le co-récepteur X4. L'utilisation de ces deux types de virus dans le modèle macaque va enfin permettre de savoir si les virus de type R5 sont exclusivement sélectionnés ou amplifiés durant la transmission virale et pendant les phases initiales de l'infection, pour évoluer ensuite vers les virus de type $\mathrm{X} 4$ ou à tropisme double X4-R5, ou si les virus de type $\mathrm{R} 5$ ou de type $\mathrm{X} 4$ sont tous les deux transmis, les virus $\mathrm{X} 4$ étant séquestrés rapidement après la transmission pour éventuellement plus tard, se répliquer plus vite que les virus de type R5.

[1. Harouse JM, et al. Science 1999; 284: 816-20.] 